\title{
Identification of Fusarium verticillioides using gene sequencing in a patient after orthotopic liver transplantation
}

Antonella Grottola ${ }^{2}$, Claudia Venturelli², William Gennari², Mauro Codeluppi', Francesca Cavrini ${ }^{3}$, Fabio Rumpianesi², Giovanni Guaraldi', Stefania Cocchi'

I Clinica delle Malattie Infettive, Dipartimento di Medicine e Specialità Mediche, Università degli Studi di Modena e R. eggio Emilia, Modena

2 Struttura Complessa di Microbiologia e Virologia, Policlinico di Modena

3 Sezione di Microbiologia, Dipartimento di Ematologia e Oncologia, Ospedale S. Orsola-Malpighi, Università degli Studi di Bologna, Bologna

Key words: Fusarium verticillioides, gene Gene sequencing, ?-Tubulin, translation Translation elongation factor EF-I

Identificazione di Fusarium verticillioides mediante sequenziamento genico in una paziente trapiantata di fegato

\section{SUMMARY}

Fusarium is an opportunistic fungal pathogen which is emerging as a significant cause of morbidity and mortality particularly in patients with haematological malignancies and bone marrow transplant recipients but also in solid organ transplant recipients.

Here we have diagnosed a case of disseminated Fusarium infection with cutaneous localization in a patient undergoing a second liver transplant after chronic rejection of primary graft.

The morphology of the isolated strain didn't allow a clear distinction between Fusarium. napiforme and Fusarium. verticillioides. For this reason it was performed a gene sequencing analysis to have a reliable identification. The isolated strain was thus identified as $F$. verticillioides

\section{INTRODUZIONE}

L'incidenza d'infezioni fungine invasive è in aumento nei pazienti immunocompromessi. Fusarium è un fungo opportunista patogeno che sta emergendo come importante causa di morbidità e mortalità soprattutto in pazienti affetti da neoplasie ematologiche, in pazienti sottoposti a trapianto di midollo osseo e occasionalmente anche in pazienti sottoposti a trapianto d'organo solido $(43,76)$. Presentiamo un caso d'infezione disseminata da Fusarium F. verticillioides con interessamento cutaneo in una paziente sottoposta a ritrapianto di fegato per rigetto cronico per il quale si è giunti all'identificazione di specie tramite l'utilizzo della tecnica di sequenziamento genico.

\section{CASO CLINICO}

Una donna romena di 30 anni veniva sottoposta a trapianto di fegato per cirrosi da colangite sclerosante primitiva. A distanza di 18 mesi, a causa di ripetuti episodi di rigetto, veniva effettuato un ri-trapianto. Dopo 23 giorni dall'ultimo intervento la paziente manifestava febbre elevata, papule di 5-10 $\mathrm{mm}$ di diametro ulcerate e dolorose sulla gamba destra di cui alcune emorragiche.

\section{MATERIALI E METODI}

\section{Esame colturale}

Da due campioni di sangue periferico, prelevati al $28^{\circ}$ giorno dopo il ritrapianto, venivano allestite emocolture. I flaconi venivano incubati nello strumento Bactec 9..240 (Becton Dickinson, Italia).

Alla comparsa di una positività dell'emocoltura erano allestiti sia un esame microscopico mediante colorazione di Gram che delle subcolture su Sabouraud dextrose agar (BioMerieuxbioMérieux, Marcy l'Etoile, France) incubate a $37^{\circ} \mathrm{C}$ e a $24^{\circ} \mathrm{C}$ per 5 settimane.

\section{Antimicogramma}

L'antimicogramma è stato eseguito utilizzando il metodo Etest per amfotericina $\mathrm{B}$, itraconazolo, fluconazolo, voriconazolo, caspofungina e seguendo le istruzioni della ditta produttrice (AB BbioMérieux, Solna, SwedenFrance). Da una coltura del ceppo clinico isolato, di 7 giorni cresciuta su potato dextrose agar a $35^{\circ} \mathrm{C}$ per 7 giorni, è stata preparata allestita una sospensione (range $0,4 \times 10^{4}-5 \times 10^{4} \mathrm{UFC} / \mathrm{ml}$ ) di conidi o sporangiospore distribuita successivamente seminata su RPMI agar $+2 \%$ glucosio (Biolife, Italiana, Milano, Italia).
In parallelo è stato allestito un analogo antimicogramma di controllo utilizzando il ceppo ATCC 22019 di Candida parapsilosis. Le piastre sono state incubate aDopo un'incubazione di 48 ore a $35^{\circ} \mathrm{C}$, la MIC è stata rilevata in corrispondenza dell'intersezione del bordo della zona ellittica di una prominente (itraconazolo, fluconazolo, voriconazolo) o di una completa (amfotericina) inibizione con la strip di antifungino. Per caspofungina è stata determinata la MEC.

\section{Sequenziamento genico}

Il DNA fungino è stato estratto dalle subcolture ottenute utilizzando il kit QIAamp DNA Mini Kit (QIAgen S.p.A., Milan, Italia) e amplificato tramite Polymerase Chain Reaction (PCR). In particolare sono stati generati due ampliconi corrispondenti ad un frammento dei geni fungini EF-1 (translation elongation factor 1-alpha ) e beta-tubulina. I prodotti di PCR per EF-1 e per la beta-tubulina sono stati ottenuti utilizzando rispettivamente i primers EF-1 e EF-2 in accordo con Bezuidenhout (32) ed i primers T1 e T22 in accordo con O'Donnell (87). Nel dettaglio la reazione di amplificazione è stata realizzata in un volume finale di 50ul contenente 100 ng di DNA estratto, 25 ul di REDTaq ReadyMix PCR Reaction Mix con $\mathrm{MgCl}_{2}$ (Sigma-Aldrich, Inc. Milano, Italia), 40 pmoli di ciascun primer.

I prodotti di amplificazione (700 bp per EF-1 e 1227 per betatubulina) sono stati sequenziati utilizzando il BigDye Terminator v3.1 Cycle Sequencing Kit e analizzati su sequenziatore automatizzato a fluorescenza ABI PRISM 3100 Analyzer (Applied Biosystems, Foster City, CA).

Le sequenze ottenute sono state analizzate con un software di allineamento (SeqScape, Applied Biosystem), ottenendo una sequenza di consenso. Tale sequenza è stata inserita in GeneBank Database dell'NIH (21) per il confronto, tramite il software BLAST, con le altre sequenze depositate al fine di ottenere l'identificazione di specie.

\section{RISULTATI}

\section{Esame colturale}

Le colture dei due campioni di sangue periferico, prelevati al $28^{\circ}$ giorno dopo il ritrapianto, risultavano positive dopo 96 ore di incubazione in Bactec 9.240 per la presenza di funghi filamentosi. L'osservazione microscopica, eseguita nelle prime tre settimane della coltura, indirizzava per Fusarium F. verticillioides; non era comunque possibile escludere Fusarium F. napiforme, specie molto simile, che differisce

\section{Corresponding author: Antonella Grottola}

Struttura Complessa di Microbiologia e Virologia Azienda Integrata Ospedaliero-Universitaria, Policlinico

Via del Pozzo, 7I - 4II00 Modena - Tel.:059 4223752 - Fax: 0594223625

E-mail: antonella.grottola@unimore.it 
per la comparsa tardiva in coltura di clamidospore e di caratteristici microconidi napiformi, secondo i criteri proposti da Guarro e Liu $(54,65)$. Così, dopo avere prolungato l'incubazione fino a 5 settimane a $24^{\circ} \mathrm{C}$, l'esame microscopico evidenziava nelle ife numerose cellule rigonfie ben strutturate che venivano scambiate per clamidospore di Fusarium $F$. napiforme (Figura I).

\section{Antimicogramma}

I risultati del test di suscettibilità sensibilità a 48 ore sono stati i seguenti: fluconazolo $\mathrm{MIC} \geq 256 \mathrm{ug} \mathrm{mg} / \mathrm{ml}$, caspofungin $\mathrm{MICMEC} \geq 32 \mathrm{ugmg} / \mathrm{ml}$, itraconazolo $\mathrm{MIC} \geq=32 \mathrm{ugmg}$ $/ \mathrm{ml}$, amfotericina B MIC $=8-16 \mathrm{ugmg} / \mathrm{ml}$, voriconazolo $\mathrm{MIC}=4 \mathrm{ugmg} / \mathrm{ml}$.(Fig. 3a e b).

In accordo con CLSI M-38 A2 è stato refertato il valore di $\mathrm{MIC} / \mathrm{MEC}$ per i farmaci antifungini testati, in quanto non sono stati finora stabiliti i relativi breakpoints interpretativi.

\section{Sequenziamento genico}

L'analisi mediante sequenziamento di un frammento del gene codificante per il Translation Elongation Factor (EF-1) e del gene codificante per la ?-tubulina permetteva d'identificare definitivamente il micete come Fusarium F. verticillioides. In particolare le sequenze del ceppo isolato mostravano un'identità del 99\%, corrispondente ad una differenza di una singola base, con il ceppo di riferimento Fusarium. verticillioides NRRL 43697 (GenBank accession number EF 453022).

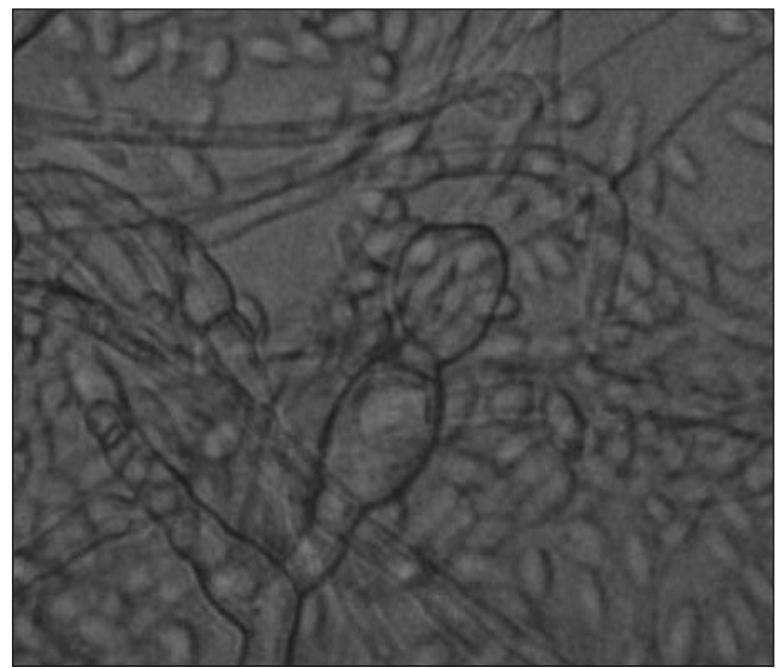

Figura I. Pseudoclamidospore o cellule rigonfie terminali e a catena (5$12 \mu \mathrm{m})$ di Fusarium verticillioides.

\section{CONCLUSIONI}

Le infezioni da Fusarium spp. tendono ad essere disseminate e la prognosi è di solito severa perché i funghi sono resistenti alla maggior parte degli agenti antifungini disponibili. Il fattore più importante per favorire 1"instaurarsi di fusariosi è la neutropenia prolungata e questo è il motivo per cui le fusariosi si osservano più frequentemente nei pazienti trapiantati di midollo rispetto ai pazienti trapiantati d'organo solido. Nel nostro caso è stata riscontrata un'a infezione dissemina- ta da Fusarium F. verticillioides con interessamento cutaneo in una paziente sottoposta a ritrapianto di fegato per rigetto cronico.

I criteri morfologici si sono rivelati insufficienti per una corretta identificazione a livello di specie del ceppo isolato; infatti l'aspetto microscopico del Fusarium indirizzava inizialmente per Fusarium F. napiforme, senza potere escludere Fusarium F.verticillioides. Solamente l'analisi mediante sequenziamento genico ha permesso dii identificare definitivamente il micete come Fusarium F. verticillioides.

In conclusione, il caso riportato sottolinea ancora una volta la complessità nell'identificazione di Fusarium species in base ai soli criteri micologici ed evidenzia la necessità di ricorrere a metodi molecolari, come il sequenziamento genico, da affiancare alle metodiche diagnostiche tradizionali.

$\mathrm{Nel}$ caso descritto il sequenziamento genico ha permesso di sciogliere il dubbio tassonomico posto dai criteri microbiologici di identificazione, ma non ha anticipato i tempi per l'inizio del trattamento antimicotico in quanto è stato eseguito dopo l'inizio della trattamento antifungino in profilassi. La refertazione dell'isolato come Fusarium. verticillioides, in associazione con l'antimicogramma, è stata fondamentale nella gestione clinica del paziente poichèé ha permesso di definire l' eziologia e di identificare la sorgente dell'evento infettivo nonchèé di impostare in modo mirato la terapia.

Da questo si evince che

la differenziazione di specie nel trattamento antimicotico permette di orientare il clinico nell'impostazione della terapia empirica basata sui dati della letteratura scientifica internazionale in attesa della disponibilità dell'antimicogramma.

Ad esempio è' noto che ceppi clinici di Fusarium solani sono resistenti alla maggior parte degli antimicotici (1): l'identificazione di specie si rivela importante per la scelta della terapia antimicotica.

In conclusione il caso riportato sottolinea la complessità nell'identificazione di Fusarium species in base ai soli criteri micologici ed evidenzia la necessità di ricorrere a metodi molecolari, come il sequenziamento genico, da affiancare alle metodiche diagnostiche tradizionali.

\section{BIBLIOGRAFIA}

1. Azor, M., J. Gené J, J. Cano J, and J. Guarro J.. 2007. Universal in vitro antifungal resistance of genetic clades of the Fusarium solani species complex. Antimicrob. Agents Chemother. 2007; 51: 1500-1503.)

2. Benson DA, Karsch-Mizrachi I, Lipman DJ, Ostell J, Wheeler DL. GenBank. Nucleic Acids Research, 2008, Jan; 36 (Database issue) D25-30).

3. Bezuidenhout CC, Prinsloo M, Van der Walt AM. Multiplex PCR-based detection of potential fumonisin-producing Fusarium in traditional african vegetables. Environ Toxicol 2006; 21: 360-366, 2006

4. Denning DW, Evans EG, Kibbler CC, Richardson MD, Roberts MM, Rogers TR et al. Guidelines for the investigation of invasive fungal infections in haematological malignancy and solid organ transplantation. British Society for Medical Mycology. Eur J Clin Microbiol Infect Dis 1997; 16 (6): 424-436

5. Guarro J, Gene J. Fusarium infections. Criteria for the identification of the responsible species. Mycoses 1992; 35 (5-6): 109-114.

6. Liu K, Howell DN, Perfect JR, Schell WA. Morphologic criteria for the preliminary identification of Fusarium, Paecilomyces, and Acremonium species by histopathology. Am J Clin Pathol 1998; 109 (1): 45-54.

7. Morrison VA, Haake RJ, Weisdorf DJ. The spectrum of non-Candida fungal infections following bone marrow transplantation. Medicine (Baltimore) 1993; 72 (2): 78-89.

8. O'Donnell K, Cigelnik E. Two divergent intragenomic rDNA ITS2 types within a monophyletic lineage of the fungus Fusarium are nonorthologous. Mol Phylogenet Evol 1997; 7 (1): 103-16. 